\title{
Exact Solutions and Conservation Laws of the Drinfel'd-Sokolov-Wilson System
}

\author{
Catherine Matjila, Ben Muatjetjeja, and Chaudry Masood Khalique \\ International Institute for Symmetry Analysis and Mathematical Modelling, Department of Mathematical Sciences, \\ North-West University, Mafikeng Campus, Private Bag X 2046, Mmabatho 2735, South Africa
}

Correspondence should be addressed to Chaudry Masood Khalique; masood.khalique@nwu.ac.za

Received 22 January 2014; Accepted 4 March 2014; Published 27 March 2014

Academic Editor: Baojian Hong

Copyright (C) 2014 Catherine Matjila et al. This is an open access article distributed under the Creative Commons Attribution License, which permits unrestricted use, distribution, and reproduction in any medium, provided the original work is properly cited.

We study the Drinfel'd-Sokolov-Wilson system, which was introduced as a model of water waves. Firstly we obtain exact solutions of this system using the $\left(G^{\prime} / G\right)$-expansion method. In addition to exact solutions we also construct conservation laws for the underlying system using Noether's approach.

\section{Introduction}

The classical Drinfel'd-Sokolov-Wilson (DSW) system given by

$$
\begin{gathered}
u_{t}+p v v_{x}=0 \\
v_{t}+q v_{x x x}+s v u_{x}+r u v_{x}=0
\end{gathered}
$$

where $p, q, r$, and $s$ are nonzero constants, has been studied by [1]. The authors obtained various types of explicit solutions for (1) by using the bifurcation method and qualitative theory of dynamical systems. Also, Yao and Li [2] and C. Liu and X. Liu [3] obtained some exact solutions for the DSW system (1) by using a direct algebra method. A special case of the classical DSW system, namely,

$$
\begin{gathered}
u_{t}+3 v v_{x}=0, \\
v_{t}+2 v_{x x x}+v u_{x}+2 u v_{x}=0,
\end{gathered}
$$

was studied by several authors [4-8]. Hirota et al. [4] investigated the soliton structure of (2) and by employing an algebraic method, Fan [5] constructed some exact solutions. By using the improved generalized Jacobi elliptic function method, Yao [6] obtained some traveling wave solutions of (2), whereas by applying the Adomian decomposition method, Inc [7] obtained approximate doubly periodic wave solutions of (2). Zhao and Zhi [8] constructed exact doubly periodic solutions of (2) by using an improved $F$-expansion method.

In this paper, we study the Drinfel'd-Sokolov-Wilson (DSW) system given by

$$
\begin{gathered}
u_{t}+\beta v v_{x}=0, \\
v_{t}+\alpha v_{x x x}+\beta v u_{x}+\beta u v_{x}=0,
\end{gathered}
$$

which can be derived from (1) by taking $p=r=s=\beta$ and $q=\alpha$. We obtain exact solutions and construct conservation laws of the DSW systems (3a) and (3b).

Nonlinear partial differential equations (PDEs) model diverse nonlinear phenomena in natural and applied sciences such as mechanics, fluid dynamics, biology, plasma physics, and mathematical finance. Therefore finding exact solutions of nonlinear PDEs is very important. Unfortunately, this is a very difficult task and there are no systematic methods that can be used to find exact solutions of the nonlinear PDEs. However, in the past few decades a number of new methods have been developed to obtain exact solutions to nonlinear PDEs. Some of these methods include the exp-function method, the homogeneous balance method, the sine-cosine method, the hyperbolic tangent function expansion method, and the $\left(G^{\prime} / G\right)$-expansion function method [9-15].

We recall that conservation laws are mathematical expressions of the physical laws, such as conservation of energy, 
mass, and momentum. They are of great significance in the solution process and reduction of PDEs. In the literature, one finds that the conservation laws have been widely used in studying the existence, uniqueness, and stability of solutions of nonlinear partial differential equations [16-18], as well as in the development and use of numerical methods [19,20]. Also, conserved vectors associated with Lie point symmetries have been employed to find exact solutions of partial differential equations [21-23]. There are various methods of constructing conservation laws. One of the methods for variational problems is by means of Noether's theorem [24]. In order to apply Noether's theorem, the knowledge of a suitable Lagrangian is necessary. For nonlinear differential equations that do not have a Lagrangian, several methods have been developed (see, e.g., [25-30]).

This paper is structured as follows. In Section 2, exact solutions of (3a) and (3b) are obtained using the $\left(G^{\prime} / G\right)$ expansion function method. In Section 3, we construct Noether's symmetries and the conserved vectors for the DSW system (3a) and (3b). Concluding remarks are presented in Section 4.

\section{Exact Solutions of the DSW System (3a) and (3b)}

In this section, we obtain exact solutions of the DSW system (3a) and (3b). We first transform the system (3a) and (3b) into a system of ordinary differential equations by using the substitutions

$$
u(x, t)=U(z), \quad v(x, t)=V(z),
$$

where $z=x-c t$. Substituting (4) into the system (3a) and (3b) and integrating with respect to $z$, we obtain the following ordinary differential equations (ODEs):

$$
\begin{aligned}
& -c U+\frac{\beta}{2} V^{2}=0, \\
& -c V+\alpha V^{\prime \prime}+\beta V U=0,
\end{aligned}
$$

where integration constants are taken to be zero. From the first equation, we obtain $U=\beta V^{2} /(2 c)$. Substituting this value of $U$ in the second equation of the system, we obtain

$$
-c V+\alpha V^{\prime \prime}+\frac{\beta^{2}}{2 c} V^{3}=0
$$

Now multiplying the above equation by $V^{\prime}$ and integrating while taking the constant of integration to be zero, we arrive at a first-order variables separable equation. Integrating this equation and reverting back to our original variables, we obtain

$$
v(x, t)=\frac{4 c^{2} \exp [\sqrt{c / \alpha}(x-c t+A)]}{1+\beta c^{2} \exp [2 \sqrt{c / \alpha}(x-c t+A)]},
$$

where $A$ is an arbitrary constant of integration. Since $U=$ $\beta V^{2} /(2 c)$, we have

$$
u(x, t)=\frac{\beta}{2 c}\left\{\frac{4 c^{2} \exp [\sqrt{c / \alpha}(x-c t+A)]}{1+\beta c^{2} \exp [2 \sqrt{c / \alpha}(x-c t+A)]}\right\}^{2}
$$

Thus, we have obtained one exact solution of the DSW system (3a) and (3b).

To obtain more exact solutions of the DSW system (3a) and (3b), we employ the $\left(G^{\prime} / G\right)$-expansion function method [15]. We assume that the solutions of the ODE (6) can be expressed as a polynomial in $\left(G^{\prime} / G\right)$ by

$$
V(z)=\sum_{i=0}^{n} \phi_{i}\left(\frac{G^{\prime}}{G}\right)^{i}
$$

where $n$ is the balancing number to be determined and the function $G(z)$ satisfies the second-order linear ODE given by

$$
G^{\prime \prime}(z)+\lambda G^{\prime}(z)+\mu G(z)=0
$$

with $\lambda$ and $\mu$ being arbitrary constants. In our case, the balancing procedure gives $n=1$. Thus

$$
V(z)=\phi_{0}+\phi_{1}\left(\frac{G^{\prime}}{G}\right) .
$$

Substituting (11) into (6) and making use of (10) and then equating all the terms with the same powers of $\left(G^{\prime} / G\right)$ to zero yield the following system of algebraic equations:

$$
\begin{gathered}
\alpha \phi_{1} \lambda^{2}+2 \alpha \phi_{1} \mu+\frac{3 \beta^{2} \phi_{0}^{2} \phi_{1}}{2 c}-c \phi_{1}=0 \\
3 \alpha \phi_{1} \lambda+\frac{3 \beta^{2} \phi_{0} \phi_{1}^{2}}{2 c}=0 \\
2 \alpha \phi_{1}+\frac{\beta^{2} \phi_{1}^{3}}{2 c}=0 \\
\alpha \phi_{1} \lambda \mu+\frac{\beta^{2} \phi_{0}^{3}}{2 c}-c \phi_{0}=0
\end{gathered}
$$

Solving the above equations, with the aid of Mathematica, we obtain

$$
\begin{gathered}
\alpha=-\frac{2 c}{\left(\lambda^{2}-4 \mu\right)}, \\
\phi_{0}=-\frac{\sqrt{2} \sqrt{c^{2}-2 \alpha c \mu}}{\beta}, \\
\phi_{0}=\frac{\sqrt{2} \sqrt{c^{2}-2 \alpha c \mu}}{\beta}, \\
\phi_{1}=\frac{2 \phi_{0}}{\lambda} .
\end{gathered}
$$

Consequently, we obtain the following two types of travelling wave solutions of the DSW system. 
For $\lambda^{2}-4 \mu>0$, we obtain the hyperbolic functions travelling wave solutions

$$
\begin{aligned}
& v_{1}(t, x) \\
& = \pm \frac{\sqrt{2\left(c^{2}-2 \alpha c \mu\right)}}{\beta} \pm \frac{2 \sqrt{2\left(c^{2}-2 \alpha c \mu\right)}}{\lambda \beta} \\
& \times\left[-\frac{\lambda}{2}+\frac{\sqrt{\lambda^{2}-4 \mu}}{2}\right. \\
& \times\left(\left(c_{1} \sinh \left(\frac{\sqrt{\lambda^{2}-4 \mu}}{2}\right)(x-c t)\right.\right. \\
& \left.+c_{2} \cosh \left(\frac{\sqrt{\lambda^{2}-4 \mu}}{2}\right)(x-c t)\right) \\
& \times\left(c_{1} \cosh \left(\frac{\sqrt{\lambda^{2}-4 \mu}}{2}\right)(x-c t)+c_{2}\right. \\
& \left.\left.\left.\times \sinh \left(\frac{\sqrt{\lambda^{2}-4 \mu}}{2}\right)(x-c t)\right)^{-1}\right)\right],
\end{aligned}
$$

$u_{1}(t, x)$

$$
\begin{gathered}
=\frac{\beta}{2 c}\left\{\begin{array}{l} 
\pm \frac{\sqrt{2\left(c^{2}-2 \alpha c \mu\right)}}{\beta} \pm \frac{2 \sqrt{2\left(c^{2}-2 \alpha c \mu\right)}}{\lambda \beta} \\
\times\left[-\frac{\lambda}{2}+\frac{\sqrt{\lambda^{2}-4 \mu}}{2}\right.
\end{array}\right.
\end{gathered}
$$$$
\times\left(\left(c_{1} \sinh \left(\frac{\sqrt{\lambda^{2}-4 \mu}}{2}\right)(x-c t)+c_{2}\right.\right.
$$$$
\times \cosh \left(\frac{\sqrt{\lambda^{2}-4 \mu}}{2}\right)(x-c t)
$$$$
\times\left(c_{1} \cosh \left(\frac{\sqrt{\lambda^{2}-4 \mu}}{2}\right)(x-c t)+c_{2}\right.
$$$$
\left.\left.\left.\left.\times \sinh \left(\frac{\sqrt{\lambda^{2}-4 \mu}}{2}\right)(x-c t)\right)^{-1}\right)\right]\right\}^{2}
$$

For $\lambda^{2}-4 \mu<0$, we obtain the trigonometric function travelling solutions

$$
\begin{aligned}
& v_{2}(t, x) \\
& = \pm \frac{\sqrt{2\left(c^{2}-2 \alpha c \mu\right)}}{\beta} \pm \frac{2 \sqrt{2\left(c^{2}-2 \alpha c \mu\right)}}{\lambda \beta} \\
& \times\left[-\frac{\lambda}{2}+\frac{\sqrt{4 \mu-\lambda^{2}}}{2}\right. \\
& \times\left(\left(-c_{1} \sin \left(\frac{\sqrt{4 \mu-\lambda^{2}}}{2}\right)(x-c t)\right.\right. \\
& +c_{2} \cos \left(\frac{\sqrt{4 \mu-\lambda^{2}}}{2}\right)(x-c t) \\
& \times\left(c_{1} \cos \left(\frac{\sqrt{4 \mu-\lambda^{2}}}{2}\right)(x-c t)+c_{2}\right. \\
& \left.\left.\left.\times \sin \left(\frac{\sqrt{4 \mu-\lambda^{2}}}{2}\right)(x-c t)\right)^{-1}\right)\right] \text {, }
\end{aligned}
$$

$u_{2}(t, x)$

$$
=\frac{\beta}{2 c}\left\{ \pm \frac{\sqrt{2\left(c^{2}-2 \alpha c \mu\right)}}{\beta} \pm \frac{2 \sqrt{2\left(c^{2}-2 \alpha c \mu\right)}}{\lambda \beta}\right.
$$$$
\times\left[-\frac{\lambda}{2}+\frac{\sqrt{4 \mu-\lambda^{2}}}{2}\right.
$$

$$
\begin{gathered}
\times\left(\left(-c_{1} \sin \left(\frac{\sqrt{4 \mu-\lambda^{2}}}{2}\right)(x-c t)+c_{2}\right.\right. \\
\left.\times \cos \left(\frac{\sqrt{4 \mu-\lambda^{2}}}{2}\right)(x-c t)\right) \\
\left.\left.\left.\left.\times\left(\frac{c_{1} \cos \left(\frac{\sqrt{4 \mu-\lambda^{2}}}{2}\right)(x-c t)+c_{2}}{2}\right)(x-c t)\right)^{-1}\right)\right]\right\}^{2} \\
\times \sin \left(\frac{\sqrt{4 \mu-\lambda^{2}}}{2} .\right.
\end{gathered}
$$




\section{Conservation Laws of the DSW Equations $(3 a)$ and $(3 b)$}

In this section, we construct the conservation laws of the DSW system (3a) and (3b). Since the third-order DSW system (3a) and (3b) does not have a Lagrangian, we cannot apply the Noether theorem. However, if we transform the third-order DSW system (3a) and (3b) to a fourth-order with the aid of the transformation $u=U_{x}, v=V_{x}$ [31], we obtain

$$
\begin{gathered}
U_{t x}+\beta V_{x} V_{x x}=0 \\
V_{t x}+\alpha V_{x x x x}+\beta V_{x} U_{x x}+\beta U_{x} V_{x x}=0 .
\end{gathered}
$$

This system has a Lagrangian given by

$$
L=\frac{1}{2}\left(\alpha V_{x x}^{2}-\beta U_{x} V_{x}^{2}-U_{x} U_{t}-V_{x} V_{t}\right)
$$

and it satisfies the Euler-Lagrange equations

$$
\frac{\delta L}{\delta U}=0, \quad \frac{\delta L}{\delta V}=0
$$

where $\delta / \delta U$ and $\delta / \delta V$ are defined by

$$
\begin{aligned}
\frac{\delta}{\delta U}= & \frac{\partial}{\partial U}-D_{t} \frac{\partial}{\partial U_{t}}-D_{x} \frac{\partial}{\partial U_{x}} \\
& +D_{t}^{2} \frac{\partial}{\partial U_{t t}}+D_{x}^{2} \frac{\partial}{\partial U_{x x}}+D_{x} D_{t} \frac{\partial}{\partial U_{t x}}-\cdots, \\
\frac{\delta}{\delta V}= & \frac{\partial}{\partial V}-D_{t} \frac{\partial}{\partial V_{t}}-D_{x} \frac{\partial}{\partial V_{x}}+D_{t}^{2} \frac{\partial}{\partial V_{t t}} \\
& +D_{x}^{2} \frac{\partial}{\partial V_{x x}}+D_{x} D_{t} \frac{\partial}{\partial V_{t x}}-\cdots,
\end{aligned}
$$

respectively.

Let us now consider the vector field

$$
\begin{aligned}
X= & \xi^{1}(t, x, U, V) \frac{\partial}{\partial t}+\xi^{2}(t, x, U, V) \frac{\partial}{\partial x} \\
& +\eta^{1}(t, x, U, V) \frac{\partial}{\partial U}+\eta^{2}(t, x, U, V) \frac{\partial}{\partial V} .
\end{aligned}
$$

The second prolongation operator, $X^{[2]}$ of $X$, is given by

$$
\begin{aligned}
X^{[2]}= & \xi^{1}(t, x, U, V) \frac{\partial}{\partial t} \\
& +\xi^{2}(t, x, U, V) \frac{\partial}{\partial x}+\eta^{1}(t, x, U, V) \frac{\partial}{\partial U} \\
& +\eta^{2}(t, x, U, V) \frac{\partial}{\partial V}+\zeta_{t}^{1} \frac{\partial}{\partial U_{t}} \\
& +\zeta_{t}^{2} \frac{\partial}{\partial V_{t}}+\zeta_{x}^{1} \frac{\partial}{\partial U_{x}}+\zeta_{x}^{2} \frac{\partial}{\partial V_{x}}+\cdots,
\end{aligned}
$$

where

$$
\begin{aligned}
\zeta_{t}^{1}= & D_{t}\left(\eta^{1}\right)-U_{t} D_{t}\left(\xi^{1}\right)-U_{x} D_{t}\left(\xi^{2}\right), \\
\zeta_{t}^{2}= & D_{t}\left(\eta^{2}\right)-V_{t} D_{t}\left(\xi^{1}\right)-V_{x} D_{t}\left(\xi^{2}\right), \\
\zeta_{x}^{1}= & D_{x}\left(\eta^{1}\right)-U_{t} D_{x}\left(\xi^{1}\right)-U_{x} D_{x}\left(\xi^{2}\right), \\
\zeta_{x}^{2}= & D_{x}\left(\eta^{2}\right)-V_{t} D_{x}\left(\xi^{1}\right)-V_{x} D_{x}\left(\xi^{2}\right), \\
D_{t}= & \frac{\partial}{\partial t}+U_{t} \frac{\partial}{\partial U}+V_{t} \frac{\partial}{\partial V}+U_{t t} \frac{\partial}{\partial U_{t}} \\
& +V_{t t} \frac{\partial}{\partial V_{t}}+U_{t x} \frac{\partial}{\partial U_{x}}+V_{t x} \frac{\partial}{\partial V_{x}}+\cdots, \\
& \frac{\partial}{\partial x}+U_{x} \frac{\partial}{\partial U}+V_{x} \frac{\partial}{\partial V}+U_{x x} \frac{\partial}{\partial U_{x}} \\
D_{x} & +V_{x x} \frac{\partial}{\partial V_{x}}+U_{t x} \frac{\partial}{\partial U_{t}}+V_{t x} \frac{\partial}{\partial V_{t}}+\cdots .
\end{aligned}
$$

We recall that $X$, given by (20), is a Noether symmetry of (16a) and (16b), if it satisfies

$$
X(L)+L\left[D_{t}\left(\xi^{1}\right)+D_{x}\left(\xi^{2}\right)\right]=D_{t}\left(B^{1}\right)+D_{x}\left(B^{2}\right),
$$

where $B^{1}(t, x, U, V)$ and $B^{2}(t, x, U, V)$ are the gauge functions. Expanding the above equation gives

$$
\begin{gathered}
-\frac{1}{2} U_{x}\left[\eta_{t}^{1}+U_{t} \eta_{U}^{1}+V_{t} \eta_{V}^{1}-U_{t} \xi_{t}^{1}\right. \\
-U_{t}^{2} \xi_{U}^{1}-U_{t} V_{t} \xi_{V}^{1} \\
\left.-U_{x} \xi_{t}^{2}-U_{t} U_{t} \xi_{U}^{2}-U_{x} V_{t} \xi_{V}^{2}\right] \\
-\frac{1}{2} V_{x}\left[\eta_{t}^{2}+U_{t} \eta_{U}^{2}+V_{t} \eta_{V}^{2}-V_{t} \xi_{t}^{1}\right. \\
-U_{t} V_{t} \xi_{U}^{1}-V_{t}^{2} \xi_{V}^{1}-V_{x} \xi_{t}^{2} \\
\left.-U_{t} V_{x} \xi_{U}^{2}-V_{t} V_{x} \xi_{V}^{2}\right] \\
-\frac{1}{2}\left(\beta V_{x}^{2}+U_{t}\right) \\
\times\left[\eta_{x}^{1}+U_{x} \eta_{U}^{1}+V_{x} \eta_{V}^{1}\right. \\
-U_{t} \xi_{x}^{1}-U_{t} U_{x} \xi_{U}^{1}-U_{t} V_{x} \xi_{V}^{1} \\
\left.-U_{x} \xi_{x}^{2}-U_{x}^{2} \xi_{U}^{2}-U_{x} V_{x} \xi_{V}^{2}\right] \\
\left.-V_{t} V_{x} \xi_{V}^{1}-V_{x} \xi_{x}^{2}-U_{x} V_{x} \xi_{U}^{2}-V_{x}^{2} \xi_{V}^{2}\right] \\
\times\left(\frac{1}{2} V_{t}+\beta U_{x} V_{x}\right) \\
\times\left[\eta_{x}^{2}+U_{x} \eta_{U}^{2}+V_{x} \eta_{V}^{2}-V_{t} \xi_{x}^{1}-U_{x} V_{t} \xi_{U}^{1}\right.
\end{gathered}
$$




$$
\begin{gathered}
+\alpha V_{x x}\left[D_{x}^{2} \eta^{2}-V_{t} D_{x}^{2} \xi^{1}-V_{x} D_{x}^{2} \xi^{2}\right. \\
-2 V_{t x}\left(\xi_{x}^{1}+U_{x} \xi_{U}^{1}+V_{x} \xi_{V}^{1}\right) \\
\left.-2 V_{x x}\left(\xi_{x}^{2}+U_{x} \xi_{U}^{2}+V_{x} \xi_{V}^{2}\right)\right] \\
+\frac{1}{2}\left[\alpha V_{x x}^{2}-\beta U_{x} V_{x}^{2}-U_{x} U_{t}-V_{x} V_{t}\right] \\
\times\left[\xi_{t}^{1}+U_{t} \xi_{U}^{1}+V_{t} \xi_{V}^{1}\right. \\
\left.+\xi_{x}^{2}+U_{x} \xi_{U}^{2}+V_{x} \xi_{V}^{2}\right] \\
=B_{t}^{1}+U_{t} B_{u}^{1}+V_{t} B_{v}^{1}+B_{x}^{2}+U_{x} B_{u}^{2}+V_{x} B_{v}^{2}
\end{gathered}
$$

This leads to an overdetermined system of PDEs for the functions $\xi^{1}, \xi^{2}, \eta^{1}, \eta^{2}, B^{1}$, and $B^{2}$. Solving the system of PDEs gives

$$
\begin{gathered}
\xi^{1}=c_{1}, \quad \xi^{2}=c_{2}, \quad \eta^{1}=f(t), \quad \eta^{2}=g(t), \\
B^{1}=A(t, x), \\
B^{2}=\frac{-1}{2} f^{\prime}(t)-\frac{1}{2} g^{\prime}(t)+H(t, x), \\
A_{t}+H_{x}=0 .
\end{gathered}
$$

We may choose $H=0$ and $A=0$ as they contribute to the trivial part of the conserved vector. The conserved vector for the second-order Lagrangian $L$ is given by $[24,31]$

$$
\begin{aligned}
T^{1}= & -B^{1}+\xi^{1} L \\
& +W^{1}\left[\frac{\partial L}{\partial U_{t}}-D_{t} \frac{\partial L}{\partial U_{t t}}-D_{x} \frac{\partial L}{\partial U_{t x}} \ldots\right] \\
& +W^{2}\left[\frac{\partial L}{\partial V_{t}}-D_{t} \frac{\partial L}{\partial V_{x t}}-D_{x} \frac{\partial L}{\partial V_{t t}} \ldots\right] \\
& +D_{t}\left(W^{1}\right) \frac{\partial L}{\partial U_{t t}}+D_{t}\left(W^{2}\right) \frac{\partial L}{\partial V_{t t}}, \\
T^{2}= & -B^{2}+\xi^{2} L \\
& +W^{1}\left[\frac{\partial L}{\partial U_{x}}-D_{t} \frac{\partial L}{\partial U_{x t}}-D_{x} \frac{\partial L}{\partial U_{x x}} \ldots\right] \\
& +W^{2}\left[\frac{\partial L}{\partial V_{x}}-D_{t} \frac{\partial L}{\partial V_{x t}}-D_{x} \frac{\partial L}{\partial V_{x x}} \ldots\right] \\
& +D_{x}\left(W^{1}\right) \frac{\partial L}{\partial U_{x x}}+D_{x}\left(W^{2}\right) \frac{\partial L}{\partial V_{x x}}
\end{aligned}
$$

where $W^{1}=\eta^{1}-U_{t} \xi^{1}-U_{x} \xi^{2}$ and $W^{2}=\eta^{2}-V_{t} \xi^{1}-V_{x} \xi^{2}$ are the characteristic functions. Now using (26) in conjunction with (25), $u=U_{x}$, and $v=V_{x}$, we obtain the following independent conserved vectors for system (3a) and (3b):

$$
\begin{aligned}
T_{1}^{1}= & \frac{1}{2}\left(\alpha v_{x}^{2}-\beta u v^{2}\right) \\
T_{1}^{2}= & -\frac{1}{2} \beta \int u_{t} d x+\frac{1}{2} \int u_{t} d x \int u_{t} d x \\
& +\beta u v \int v_{t} d x+\frac{1}{2} \int v_{t} d x \int v_{t} d x \\
& +\alpha u_{x x} \int v_{t} d x-\alpha v_{t} v_{x} \\
T_{2}^{1}= & \frac{1}{2}\left(u^{2}+v^{2}\right) \\
T_{2}^{2}= & -\frac{1}{2} \alpha v_{x}^{2}+\alpha v v_{x x}+\beta u v^{2}
\end{aligned}
$$

We note that (27) is a nonlocal conserved vector, whereas (28) is a local conserved vectors. Also, for the arbitrary functions $f(t)$ and $g(t)$, we obtain the following conserved vectors:

$$
\begin{aligned}
T_{(f, g)}^{1}= & -\frac{1}{2} u f(t)-\frac{1}{2} v g(t), \\
T_{(f, g)}^{2}= & \frac{1}{2} f^{\prime}(t) \int u d x+\frac{1}{2} g^{\prime}(t) \int v d x \\
& +f(t)\left[-\frac{1}{2} \beta v^{2}-\frac{1}{2} \int u_{t} d x\right] \\
& +g(t)\left[-\frac{1}{2} \beta u v-\frac{1}{2} \int v_{t} d x-\alpha v_{x x}\right],
\end{aligned}
$$

which gives us infinitely many nonlocal conservation laws.

\section{Conclusion}

The third-order DSW system (3a) and (3b) was studied. Exact solutions of the DSW system were obtained using direct integration and the $\left(G^{\prime} / G\right)$-expansion function method. The solutions obtained were hyperbolic and trigonometric solutions. In addition conservation laws were also derived. This system does not have a Lagrangian. In order to invoke Noether's theorem we used the transformations $u=U_{x}$ and $v=V_{x}$ to convert the DSW system to a fourth-order system, which has a Lagrangian. The conservation laws were then obtained and consisted of a local and infinite number of nonlocal conserved vectors.

\section{Conflict of Interests}

The authors declare that there is no conflict of interests regarding the publication of this paper.

\section{Acknowledgments}

All authors would like to thank NRF and North-West University, Mafikeng Campus, for financial support. 


\section{References}

[1] Z. Wen, Z. Liu, and M. Song, "New exact solutions for the classical Drinfel'd-Sokolov-Wilson equation," Applied Mathematics and Computation, vol. 215, no. 6, pp. 2349-2358, 2009.

[2] R.-x. Yao and Z.-b. Li, "New exact solutions for three nonlinear evolution equations," Physics Letters A, vol. 297, no. 3-4, pp. 196204, 2002.

[3] C. Liu and X. Liu, "Exact solutions of the classical Drinfel'dSokolov-Wilson equations and the relations among the solutions," Physics Letters A, vol. 303, no. 2-3, pp. 197-203, 2002.

[4] R. Hirota, B. Grammaticos, and A. Ramani, "Soliton structure of the Drinfel'd-Sokolov-Wilson equation," Journal of Mathematical Physics, vol. 27, no. 6, pp. 1499-1505, 1986.

[5] E. Fan, "An algebraic method for finding a series of exact solutions to integrable and nonintegrable nonlinear evolution equations," Journal of Physics A: Mathematical and General, vol. 36, no. 25, pp. 7009-7026, 2003.

[6] Y. Yao, "Abundant families of new traveling wave solutions for the coupled Drinfel'd-Sokolov-Wilson equation," Chaos, Solitons \& Fractals, vol. 24, no. 1, pp. 301-307, 2005.

[7] M. Inc, "On numerical doubly periodic wave solutions of the coupled Drinfel'd-Sokolov-Wilson equation by the decomposition method," Applied Mathematics and Computation, vol. 172, no. 1, pp. 421-430, 2006.

[8] X.-Q. Zhao and H.-Y. Zhi, "An improved F-expansion method and its application to coupled Drinfel'd-Sokolov-Wilson equation," Communications in Theoretical Physics, vol. 50, no. 2, pp. 309-314, 2008.

[9] J.-H. He and X.-H. Wu, "Exp-function method for nonlinear wave equations," Chaos, Solitons \& Fractals, vol. 30, no. 3, pp. 700-708, 2006

[10] Z. Y. Yan and H. Q. Zhang, "On a new algorithm of constructing solitary wave solutions for systems of nonlinear evolution equations in mathematical physics," Applied Mathematics and Mechanics, vol. 21, no. 4, pp. 383-388, 2000.

[11] M. L. Wang, "Solitary wave solutions for variant Boussinesq equations," Physics Letters A, vol. 199, no. 3-4, pp. 169-172, 1995.

[12] A. M. Wazwaz, "Compactons and solitary patterns structures for variants of the KdV and the KP equations," Applied Mathematics and Computation, vol. 139, no. 1, pp. 37-54, 2003.

[13] E. Fan, "Extended tanh-function method and its applications to nonlinear equations," Physics Letters A, vol. 277, no. 4-5, pp. 212-218, 2000.

[14] A. M. Wazwaz, "The tanh method for compact and noncompact solutions for variants of the KdV-Burger and the $K(n, n)$-Burger equations," Physica D, vol. 213, no. 2, pp. 147-151, 2006.

[15] M. Wang, X. Li, and J. Zhang, "The $\left(G^{\prime} / G\right)$-expansion method and travelling wave solutions of nonlinear evolution equations in mathematical physics," Physics Letters A, vol. 372, no. 4, pp. 417-423, 2008.

[16] P. D. Lax, "Integrals of nonlinear equations of evolution and solitary waves," Communications on Pure and Applied Mathematics, vol. 21, pp. 467-490, 1968.

[17] T. B. Benjamin, "The stability of solitary waves," Proceedings of the Royal Society A: Mathematical, Physical and Engineering Sciences, vol. 328, pp. 153-183, 1972.

[18] R. J. Knops and C. A. Stuart, "Quasiconvexity and uniqueness of equilibrium solutions in nonlinear elasticity," Archive for Rational Mechanics and Analysis, vol. 86, no. 3, pp. 233-249, 1984.
[19] R. J. LeVeque, Numerical Methods for Conservation Laws, Birkhäuser, Basel, Switzerland, 1992.

[20] E. Godlewski and P.-A. Raviart, Numerical Approximation of Hyperbolic Systems of Conservation Laws, Springer, Berlin, Germany, 1996.

[21] A. Sjöberg, "Double reduction of PDEs from the association of symmetries with conservation laws with applications," Applied Mathematics and Computation, vol. 184, no. 2, pp. 608-616, 2007.

[22] A. H. Bokhari, A. Y. Al-Dweik, A. H. Kara, F. M. Mahomed, and F. D. Zaman, "Double reduction of a nonlinear $(2+1)$ wave equation via conservation laws," Communications in Nonlinear Science and Numerical Simulation, vol. 16, no. 3, pp. 1244-1253, 2011.

[23] G. L. Caraffini and M. Galvani, "Symmetries and exact solutions via conservation laws for some partial differential equations of mathematical physics," Applied Mathematics and Computation, vol. 219, no. 4, pp. 1474-1484, 2012.

[24] E. Noether, "Invariante variationsprobleme," Nachrichten von der Königlichen Gesellschaft der Wissenschaften zu G öttingen, Mathematisch-Physikalische Klasse, Heft, vol. 2, pp. 235-257, 1918.

[25] P. S. Laplace, Traite de Mecanique Celeste, vol. 1, Paris, France, 1978, (English translation Celestial Mechanics, New York, NY, USA, 1966).

[26] H. Steudel, "Über die Zuordnung zwischen Invarianzeigenschaften und Erhaltungssätzen," Zeitschrift für Naturforschung, vol. 17a, pp. 129-132, 1962.

[27] P. J. Olver, Applications of Lie Groups to Differential Equations, Springer, New York, NY, USA, 1993.

[28] S. C. Anco and G. Bluman, "Direct construction method for conservation laws of partial differential equations. I. Examples of conservation law classifications," European Journal of Applied Mathematics, vol. 13, no. 5, pp. 545-566, 2002.

[29] A. H. Kara and F. M. Mahomed, "Relationship between symmetries and conservation laws," International Journal of Theoretical Physics, vol. 39, no. 1, pp. 23-40, 2000.

[30] A. H. Kara and F. M. Mahomed, "Noether-type symmetries and conservation laws via partial Lagrangians," Nonlinear Dynamics, vol. 45, no. 3-4, pp. 367-383, 2006.

[31] R. Naz, F. M. Mahomed, and T. Hayat, "Conservation laws for third-order variant Boussinesq system," Applied Mathematics Letters, vol. 23, no. 8, pp. 883-886, 2010. 


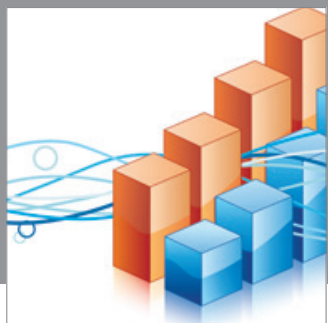

Advances in

Operations Research

mansans

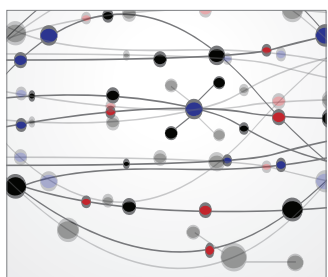

The Scientific World Journal
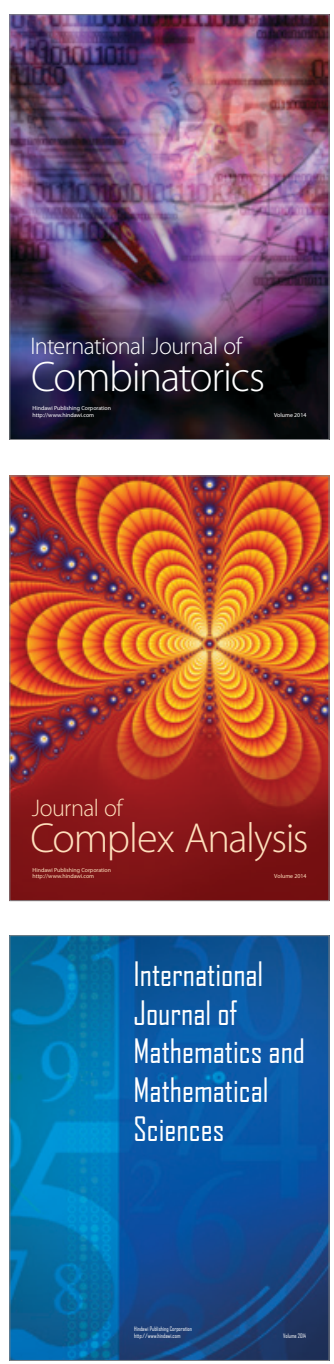
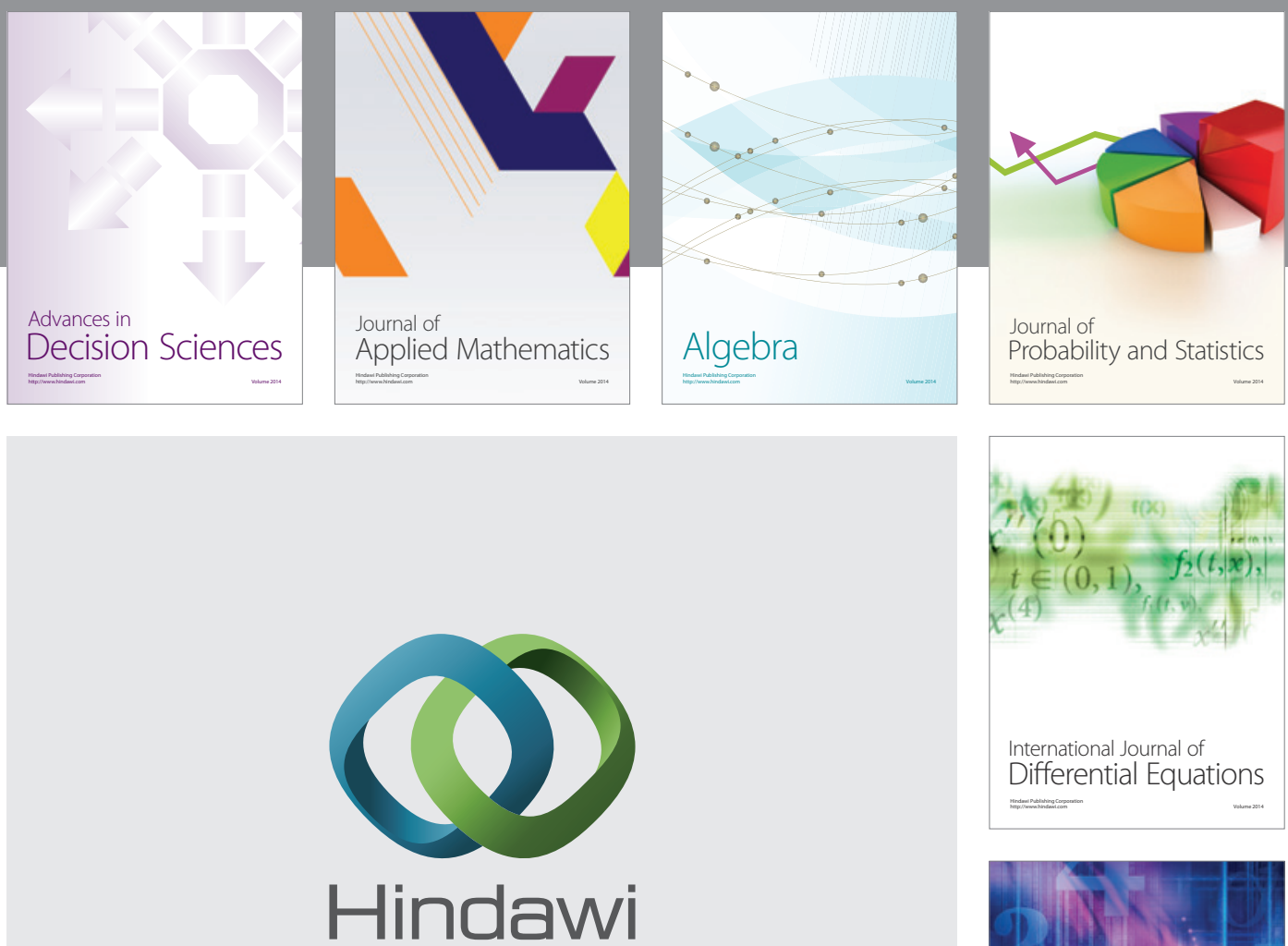

Submit your manuscripts at http://www.hindawi.com
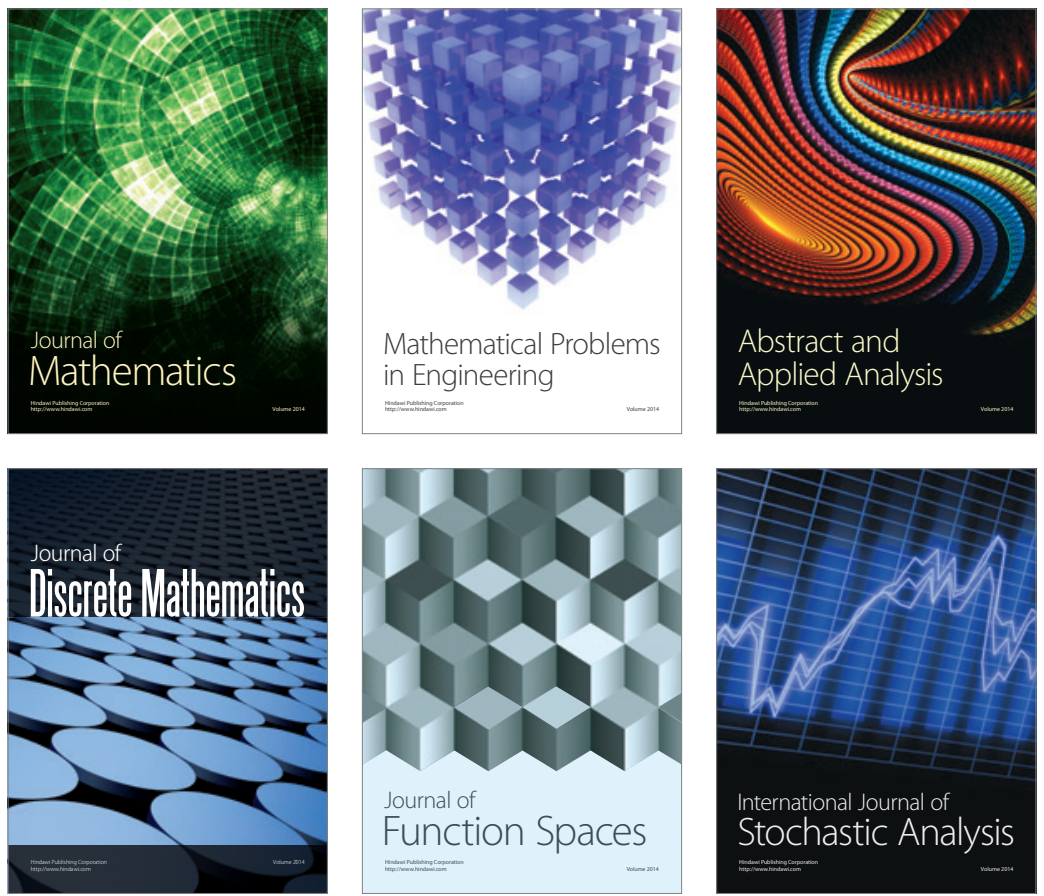

Journal of

Function Spaces

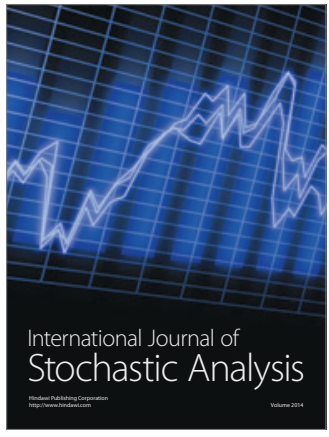

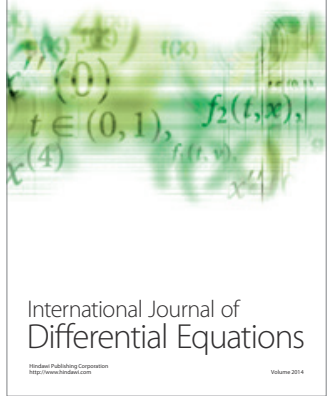
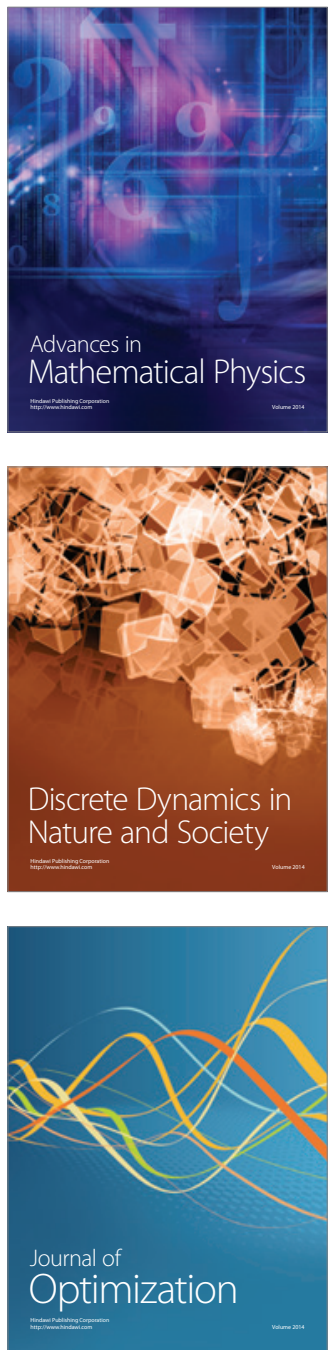